\title{
Effect of Endogenous Faecal Glucocorticoid Metabolites on Reproduction in Wild Pigs - A Non Invasive Approach
}

Boon Allwin ${ }^{1^{*}}$, Jayathangaraj $\mathbf{M G}^{1}$, Palanivelrajan $\mathbf{M}^{1}$ and Raman $\mathbf{M}^{2}$

${ }^{1}$ Department of Wildlife Science, Madras Veterinary College, Chennai, Tanuvas, Tamil Nadu, India

${ }^{2}$ TRVPB, TANUVAS, Chennai, Tamil Nadu, India

"Corresponding author: Boon Allwin, Department of Wildlife Science, Madras Veterinary College, Chennai, Tanuvas, Tamil Nadu, India, Tel: 914425304000 ; E-mail: boonallwin@gmail.com

Received date: Nov 15, 2015; Accepted date: Feb 27, 2016; Published date: Feb 29, 2016

Copyright: ( 2016 Allwin B, et al. This is an open-access article distributed under the terms of the Creative Commons Attribution License, which permits unrestricted use, distribution, and reproduction in any medium, provided the original author and source are credited.

\begin{abstract}
Wild pigs are found to be the most prolific, very intelligent and secretive. These animals are adaptive ecological generalists and survive in wide range of habitats worldwide. The reproductive traits of wild pigs are extraordinary showing high prolificacy. Corticosteroids can exert inhibitory and facilitory effects on reproduction. In order to assess the attributable stress on reproduction a non-invasive method of assessing faecal glucocorticoid metabolites was applied in this study. Samples from wild pigs from three different regions were collected and were subjected to Enzyme Linked Imuno Sorbent Assay (ELISA). The purpose of this paper is to discuss the effect of stress on reproduction in wild pigs. Corticosteroids may act at the level of the ovary to suppress aromatase activity and thereby reduce the elevated circulating estrogens considered by many to be responsible for hyper secretion of Lutenizing Hormone (LH). Interestingly the acute level of stress is found to be facilitory and the chronic found to be inhibitory.
\end{abstract}

Keywords: Corticosteroids; Reproduction; Stress; Wild pigs

\section{Introduction}

Wild pig (Sus scrofa) populations have been reported to fluctuate greatly in size. Such changes are due to interactive variations in climatic, anthropogenic, epidemic and, to a certain degree, biocentric factors [1]. Such external influences can result in high levels of mortality being realized within a wild pig population. However, because of their high reproductive potential, wild pig populations are resilient with the ability to rapidly recover from such dramatic reductions. Because of this, wild pig population sizes can oscillate widely over the short term, being more responsive/reactive to these external forces rather than multiannual cyclic in nature. Understanding the population demographics and dynamics of this invasive species is critical to being able to successfully manage or control local numbers of these animals.

The population biology of wild pigs is unique among most large mammals. Having a high reproductive potential with a concurrent high mortality rate caused population of these animals to fluctuate in response to a combination of several factors (e.g., density, food availability, severe weather conditions). The variability of the demographics (e.g., sex ratio, age class composition, mean lifespan) and other parameters (e.g., density, rate of increase, dispersal) of these populations reflects these broad oscillations in size. These stressors lead to corticosteroid release that is responsible for the regulation of population. Adult females have a 21-day oestrus cycle, with a gestation period of about 111-114 days, producing a litter of 4-10 piglets, depending on the sow's age, weight and nutritional balance.

It appears that the inhibitory action of corticosteroids on reproduction is exerted at higher brain centers, including the hypothalamus, as well as at the anterior pituitary. Corticosteroid receptors (predominantly type II) are present in the hypothalamus and anterior pituitary. Immuno histochemical studies have further localized corticosteroid receptors in neuropeptide $\mathrm{Y}$ and catecholamine neurons in the hypothalamus and in corticotrophs in the anterior pituitary [2]. Corticosteroid receptors have also been identified in ovarian granulosa cells. Other endocrine tissues demonstrated to possess corticosteroid receptors include the placenta and interstitial cells in the testes. Corticosteroid receptors are strategically located along the reproductive axis and thus are in a position to regulate endocrine/reproductive function.

\section{Materials and Methods}

\section{The study area}

Study on faecal cortisol and in wild pigs (Sus scrofa) interfering with agriculture was carried out in areas adjoining the Western Ghats (Mudumalai tiger reserve, Anamalai tiger reserve) and Eastern Ghats (Sathyamangalam region) of Tamil Nadu state in India during November, 2013 to February, 2014. The population was counted by direct sighting method using their field signs [3] during the start of the period and till the end.

\section{Number of samples examined}

Fresh faecal samples were obtained from free-ranging wild pigs in agricultural fields of adjoining forest regions of Mudumalai $(n=10)$, Sathyamangalam $(n=10)$ and Anaimalai $(n=10)$ hypothesizing that the animals that wandered in the fringes are the highly stressed Individuals. Throughout this study period, faecal samples were collected subsequent to the thorough mixing of the freshly voided fecal materials and were stored in 80 per cent methanol for steroid extraction pertaining to the estimation of cortisol by ELISA validated by Allwin et al. [4]. Well-mixed wet feces (0.6 gram) was placed in a 
Page 2 of 5

capped tube, containing $2.00 \mathrm{ml} 80$ per cent methanol, vortexed for 30 minutes and then the tubes were carefully centrifuged for 20 minutes at $2500 \mathrm{rpm}$. The supernatant material was diluted in Phosphate Buffer Saline and stored at $-80^{\circ} \mathrm{C}$ for subsequent use. Cortisol estimation was done using the ELISA KIT-DSI-EIA. The calibration curve with the mean absorbance on Y-axis and the calibrator concentration on X-axis was obtained using a 4-parameter curve by immuno assay software. The value of cortisol concentration of the unknowns was read directly from the calibration curve (Figure 1). The statistical analysis of the data was carried out as per the guidelines furnished by Snedecor and Cochren [5], using one way ANOVA.

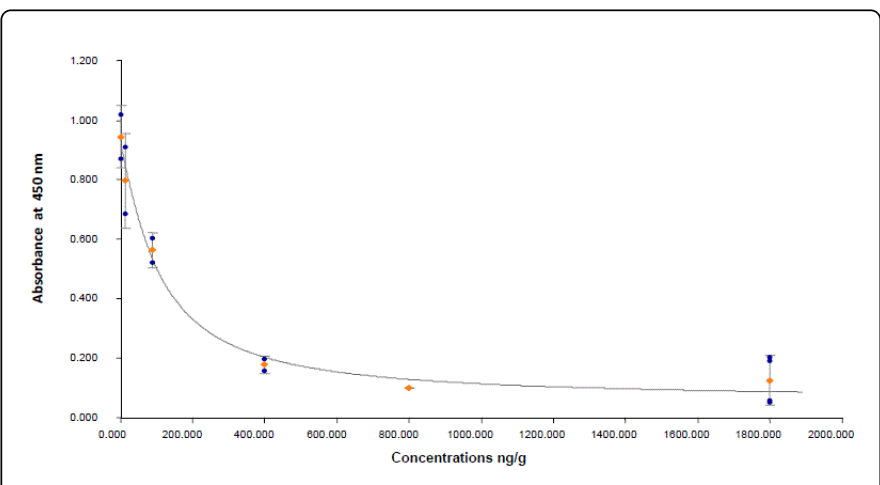

Figure 1: Standard Curve-Faecal Cortisol.

\section{Results}

\section{Faecal cortisol of wild pigs}

The faecal samples of wild pigs obtained from adjoining areas of Mudumalai, Sathyamangalam and Anaimalai wildlife regions were processed and subsequently subjected to estimation of cortisol concentration by using the ELISA kit (DSI-EIA- STEROIDCORTISOL EHE-151). Using the ELISA reader, the absorbance values of standards as well as the samples were analyzed and standard curve was obtained using standard techniques.

On Comparison of mean faecal cortisol among wild pigs of adjoining regions, the statistical analysis revealed absence of variations between the wild pigs of all the three different adjoining areas (Table 1) that proved that the stress was uniform in all these areas concerned. The mean over all faecal cortisol level in wild pigs $(n=30)$ was found to be $302.99 \mathrm{ng} / \mathrm{g}$. The mean cortisol concentrations in the faecal samples obtained from the wild pigs, between the adjoining areas of Western Ghats (Mudumalai and Anaimalai regions) and Eastern Ghats (Sathyamangalam region). It was estimated that the wild pigs of the Western Ghats showed a higher level of faecal cortisol concentration $342.70 \mathrm{ng} / \mathrm{g}$ than that of the Eastern Ghats $223.57 \mathrm{ng} / \mathrm{g}$ of faeces. The population of wild pigs also drastically reduced from the start period November, 2013 to February, 2014, it is supposed to be noted that the study was based at the start of winter which is usually drastic in these regions study area.

\begin{tabular}{|l|l|l|l|}
\hline S No & $\begin{array}{l}\text { Adjoining } \\
\begin{array}{l}\text { Regions } \\
\text { Mudumalai } \\
(\mathbf{n}=10)\end{array}\end{array}$ & $\begin{array}{l}\text { Adjoining } \\
\text { Regions } \\
\text { Sathyamangalam } \\
(\mathbf{n}=10)\end{array}$ & $\begin{array}{l}\text { Adjoining } \\
\text { Regions } \\
\text { Anaimalai } \\
(\mathbf{n}=10)\end{array}$ \\
\hline 1 & 175.79 & 141.81 & 245.63 \\
\hline
\end{tabular}

\begin{tabular}{|l|l|l|l|}
\hline 2 & 575.66 & 256.83 & 201.91 \\
\hline 3 & 579.39 & 413.42 & 464.56 \\
\hline 4 & 322.25 & 254.75 & 245.68 \\
\hline 5 & 227.94 & 146.18 & 235.87 \\
\hline 6 & 225.95 & 277.92 & 241.01 \\
\hline 7 & 684.37 & 154.85 & 306.56 \\
\hline 8 & 178.74 & 265.77 & 515.43 \\
\hline 9 & 276.47 & 148.12 & 397.33 \\
\hline 10 & 247.52 & 175.98 & 506.30 \\
\hline Mean & $349.41 \pm 59.81$ & $223.57 \pm 27.53$ & $336.03 \pm 38.83$ \\
\hline & F Value & & $2.453 \mathrm{NS}$ \\
\hline
\end{tabular}

Table 1: Faecal Cortisol Level in Wild Pigs (ng/g).

\section{Discussion}

The mean faecal cortisol level in each of the adjoining areas of Mudumalai, Sathyamangalam and Anaimalai wildlife regions were revealed in Table 1 in addition to the cortisol values of each sample from these pigs. The mean faecal cortisol levels were higher in wild pigs and the variations in stress levels due to the existence of multifaceted etiological factors including the differences in the management related measures might be assigned as the causal factors for encountering of such differences in the levels of faecal cortisol concentrations pertaining to the wild pigs. The overall mean cortisol level was found to be $302.99 \mathrm{ng} / \mathrm{g}$ in case of wild pigs. During the statistical analysis (Table 1), in this study, the mean faecal cortisol concentration in wild pigs was found to be higher it was found to reveal no significant variations $(P \leq 0.01)$, in the adjoining areas of Mudumalai, Sathyamangalam and Anaimalai wildlife regions meaning the level stress was the same pertaining to any wildlife region. The existence of such highly significant variations in terms of increase of faecal cortisol level in wild pigs might be attributed to the various biotic as well as abiotic factors, like reduced availability of feed materials including water for drinking, adverse change in the environmental conditions, proximity of various species of predators and visitors agonistic encounters social challenges, lack of highly palatable and easily available feed resources etc. The increased faecal cortisol level as encountered in wild pigs of this study might be due to the stress factors operating on this species. This was in agreement with the report presented by Touma and Palme [6] who opined that disturbances caused by the presence of humans, agonistic encounters, social challenges etc. might lead to the influence of faecal glucocorticoid metabolite in various species of mammals. In this regard, it becomes noteworthy to mention the report furnished by Pride [7] who quoted that glucocorticoid measures could be useful predictors of individual survival probabilities in the wild populations and existence of high glucocorticoid levels indicated the lowered individual fitness or even population variability. Mateo [8] opined that elevation of cortisol observed at emergence might facilitate the acquisition of anti-predator behaviors. The encountering of elevated level of faecal cortisol concentrations in majority of individual wild pigs indicated the existence of stress causing factors pertaining to the wild pigs belonging to Mudumalai, Sathyamangalam and Anaimalai 
wild life regions. Hence it could logically assumed that the wild pigs get involved in human-animal conflicts by interfering the agriculture field developed by farming community inhabiting the immediate adjoining areas of these three wildlife regions. Though it might be difficult to say whether it was acute stress or chronic stress that operated in the wild pigs under study, it becomes noteworthy to mention the report furnished by Schwarzenberger et al. [9] who stated that the delayed between the circulation of steroids and their appearance in urine samples was rather short but the lag time of faecal steroids was about 12-24 hours in ruminants and about 24 hours to over 48 hours in animals that were hind gut fermentors like horse, elephants, pigs, rhinoceros and primates. Due to the encountering of enhanced faecal cortisol level in the faecal samples of majority of the individual wild pigs, it might be assumed that the nature of stress factors related with such an elevation might probably be a chronically existing stress than the acute type of stress. Further variations in the different types of habitat, meteorological factors etc. might be assigned as the causal factors for the existence of chronic type of stress as revealed by enhanced faecal cortisol concentration in majority of wild pigs.

Stress has been demonstrated to exert both inhibitory and facilitory effects on reproductive function (Figure 2) the divergent effects can be explained or the basis of two variables:

1) Length of exposure, and

2) Background of estrogen priming.

Acute exposure generally facilitates whereas chronic exposure normally inhibits reproductive function. The growth and decline of animal populations in the wild resulted in part from endocrine responses to population density. During peak density years, stress from overcrowding was suggested to cause hyper secretion of ACTH and adrenal steroids, which results in inhibition of reproductive function and a resultant down-regulation of population growth and expansion. Since this early suggestion, many studies have supported the concept that chronic stress with or without adrenal involvement and chronic activation of the adrenal can indeed inhibit reproductive function. Chronic stress has been shown to suppress luteinizing hormone (LH) and testosterone secretion in adult male rats, inhibit sexual maturation in both male and female rats, and impair normal pregnancy resulting in decreased litter and offspring size [10,11]. It is well known that estradiol can change the sensitivity of the anterior pituitary to GnRH; this effect may explain the divergent effects of stress on LH seen in low vs. high estrogen states [12].

\section{Role of the adrenal in the regulation of reproductive function}

The action of stress or ACTH appeared to be exerted largely through adrenal steroids. However, adrenalectomy before day 25 delays puberty in the female rat, an effect that can be reversed by corticosterone replacement $[13,14]$. This delay in puberty may result from reduced FSH secretion as adrenalectomy in immature female rats has been reported to cause an acute suppression of FSH levels. Adrenalectomy in the adult cycling rat results in attenuation of preovulatory gonadotropin secretion, inhibition of follicular development and ovulation number, increased irregular cyclicity, and a prolonged critical period for LH release [14]. Furthermore, many studies have demonstrated that, in contrast to the inhibitory effects of chronic activation of the ACTH adrenal axis, acute activation is often found to actually facilitate reproductive function.

\section{Physiological role of adrenal steroids in reproduction}

A prominent role has been given to the ovary as the source of steroids in regulation of $\mathrm{LH}$ and FSH secretion. However, the adrenal also has great capacity to secrete steroids (progesterone, and corticosterone) and may contribute significantly to the overall steroid bank leading to preovulatory surges of $\mathrm{LH}$ and $\mathrm{FSH}$, the estrous surge of FSH, and ovulation in the female. As estrogen levels are high on proestrus, elevation of adrenal progesterone and corticosteroids could participate in initiating the preovulatory LH and FSH surge. If, as suggested previously, rising adrenal steroids initiate release of LH and FSH, which serves to stimulate secretion of ovarian steroids (progesterone), which in turn evokes still greater release of LH and FSH, ensuring complete ovulatory activity, then one would expect the whole process to be interrupted if the circadian adrenal rhythm were abolished. Therefore, it would appear that adrenal progesterone and corticosteroids may exert an important role on proestrus to initiate and synchronize the LH and FSH surge.

Corticosteroids have consistently been shown to selectively release FSH in vivo and in vitro. Corticosterone, cortisol, have the ability to also increase basal FSH release the morning after administration to estrogen-primed ovariectomized rats. These findings may have relevance with respect to the secondary surge of FSH that occurs on estrus, especially as serum corticosterone levels are elevated throughout the afternoon of proestrus. A selective decrease in FSH levels and disruption of follicle development has been reported to follow adrenalectomy. Thus, the selective release of FSH by corticosteroids may be one of the mechanisms that could explain the ability of corticosterone replacement to reverse the inhibitory effects of adrenalectomy on sexual maturation [11] explaining the population stasis at any point.

Corticosteroids have been demonstrated to exert direct effects at the level of the ovary. Corticosteroids have been shown to inhibit aromatase activity while they stimulate progesterone release by granulosa cells in vitro [15]. Perhaps more intriguing are findings that corticosteroids stimulate tissue plasminogen activator (TPA) activity in granulosa cells [16]. As TPA is thought to play an important role in ovulation, this effect could have important physiological relevance. This finding may also explain that dexamethasone treatment on proestrus, in some cases resulted in an unexpectedly large number of eggs per ovulating animal $[17,18]$. 
Citation: Allwin B, Jayathangaraj MG, Palanivelrajan M, Raman M (2016) Effect of Endogenous Faecal Glucocorticoid Metabolites on Reproduction in Wild Pigs - A Non Invasive Approach. J Veterinar Sci Technol 7: 307. doi:10.4172/2157-7579.1000307

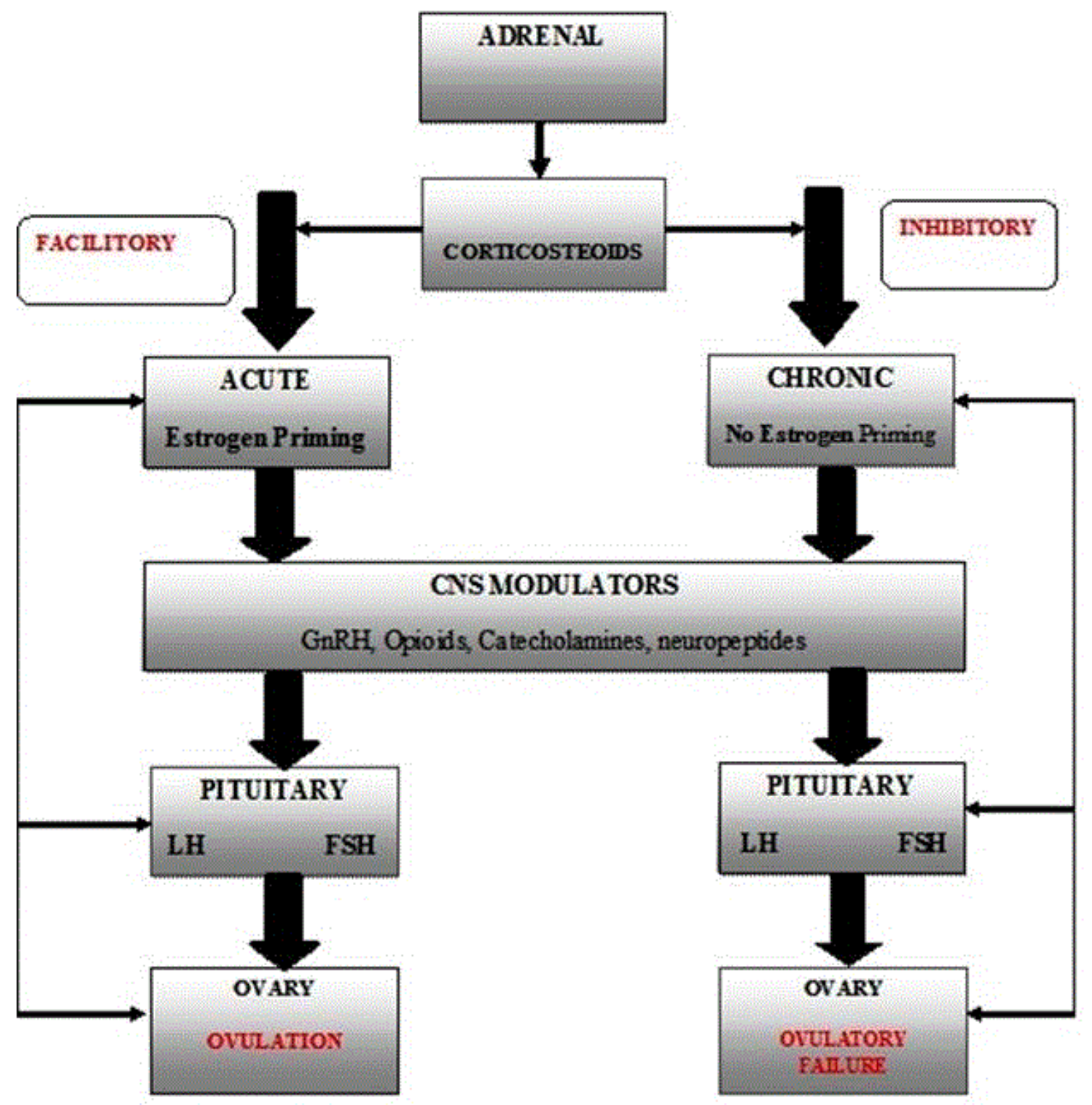

Figure 2: Inhibitory and Facilitory Effects on Reproductive Function.

\section{Conclusion}

Thus depending on the type of exposure either acute or chronic, the effect of endogenous cortisol becomes facilitory or inhibitory respectively pertaining to the indwelling reproductive capabilities of the wild pigs. Acute stress, stimulates LH release, enhances fertility, and enhances ovulatory activity. Chronic stress, inhibits LH secretion, inhibits fertility, cyclicity, follicle Development and inhibits sexual maturation, Impairs pregnancy and decreases offspring size. This phenomenon explains the effective management of wild populations by stress, naturally placing things perfectly at balance in the ecological relationship owning to the population performance and reproduction. However intense studies at the core areas are to be taken to further come to concrete conclusions on their reproduction dynamics.

\section{References}

1. Lozan M (1995) Factors that limit the number of wild boars (Sus scrofa) in the Republic of Moldova. Journal of Mountain Ecology (Ibex) 3: 211.

2. Härfstrand A, Fuxe K, Cintra A, Agnati LF, Zini I, et al. (1986) Glucocorticoid receptor immunoreactivity in monoaminergic neurons of rat brain. Proc Natl Acad Sci USA 83: 9779-9783.

3. Allwin B, Jayathangaraj MG, Palanivelrajan M, Raman M (2015) Enumerating endogenous faecal glucocorticoid metabolites as indicators of stress in wild pigs interfering with agriculture adjoining forest regions correlating with conflict and meteorological factors - A Non invasive approach. International Journal of advanced Multidisciplinary Research 2: 63-76.

4. Allwin B, Jayathangaraj MG, Palanivelrajan M, Raman M (2015) Wild Pig Field Signs - Identity and Confirmation. International Journal of Life Sciences Research 3: 205-213. 
Citation: Allwin B, Jayathangaraj MG, Palanivelrajan M, Raman M (2016) Effect of Endogenous Faecal Glucocorticoid Metabolites on Reproduction in Wild Pigs - A Non Invasive Approach. J Veterinar Sci Technol 7: 307. doi:10.4172/2157-7579.1000307

Page 5 of 5

5. Snedecor GW, Cochran WC (1989) Statistical Methods. (8th Edn) Iowa State University Press, Ames, IA, USA.

6. Touma C, Palme R (2005) Measuring fecal glucocorticoid metabolites in mammals and birds: the importance of validation. Ann NY Acad Sci 1046: 54-74.

7. Ethan Pride R (2005) High faecal glucocorticoid levels predict mortality in ring-tailed lemurs (Lemur catta). Biol Lett 1: 60-63.

8. Mateo JM (2006) Developmental and geographic variation in stress hormones in wild Belding's ground squirrels (Spermophilus beldingi). Horm Behav 50: 718-725.

9. Schwarzenberger F, Mostl E, Palme R, Bamberg E (1996) Faecal steroid analysis for non-invasive monitoring of reproductive status in farm, wild and zoo animals. Animal Reproduction Science 42: 515-526.

10. Christian JJ, Lemunyan CD (1958) Adverse effects of crowding on lactation and reproduction of mice and two generations of their progeny. Endocrinology 63: 517-529.

11. Ramaley JA (1974) Adrenal-gonadal interactions at puberty. Life Sci 14 1623-1633.

12. Brann DW, Putnam CD, Mahesh VB (1990) Corticosteroid regulation of gonadotropin and prolactin secretion in the rat. Endocrinology 126: 159-166.
13. Macfarland LA, Mann DR (1977) The inhibitory effects of ACTH and adrenalectomy on reproductive maturation in female rats. Biol Reprod 16: 306-314.

14. Mann DR, Korowitz CD, Barraclough CA (1975) Adrenal gland involvement in synchronizing the preovulatory release of LH in rats. Proc Soc Exp Biol Med 150: 115-120.

15. Hsueh AJ, Erickson GF (1978) Glucocorticoid inhibition of FSH-induced estrogen production in cultured rat granulosa cells. Steroids 32: 639-648.

16. Wang C, Leung A (1989) Glucocorticoids stimulate plasminogen activator production by rat granulosa cells. Endocrinology 124: 1595-1601.

17. De Greef WJ, Van der Schoot P (1987) Effects of dexamethasone on ovarian activity in rats. Acta Endocrinol (Copenh) 116: 350-356.

18. Kamel F, Kubajak CL (1987) Modulation of gonadotropin secretion by corticosterone: interaction with gonadal steroids and mechanism of action. Endocrinology 121: 561-568. 